\title{
An infrared study of local galaxy mergers
}

\author{
A. Carpineti ${ }^{1}$, S. Kaviraj ${ }^{2,1,3}$, A. K. Hyde ${ }^{1}$, D. L. Clements ${ }^{1}$, K. Schawinski ${ }^{5}$, D. Darg ${ }^{3}$, and C. J. Lintott St, $^{3,4}$ \\ 1 Blackett Laboratory, Imperial College London, London SW7 2AZ, UK \\ e-mail: alfredo.carpineti07@imperial.ac.uk \\ 2 University of Hertfordshire, Hatfield, Hertfordshire, AL10 9AB, UK \\ 3 Department of Physics, University of Oxford, Keble Road, Oxford OX1 3RH, UK \\ 4 Adler Planetarium, 1300 S. Lakeshore Drive, Chicago, IL 60605, USA \\ 5 Institute for Astronomy, Department of Physics, ETH Zurich, Wolfgang-Pauli-Strasse 16, 8093 Zurich, Switzerland
}

Received 4 November 2014 / Accepted 16 March 2015

\begin{abstract}
We combine a large, homogeneous sample of $\sim 3000$ local mergers with the Imperial IRAS Faint Source Redshift Catalogue (IIFSCz), to perform a blind far-infrared (FIR) study of the local merger population. The IRAS-detected mergers are mostly (98\%) spiral-spiral systems, residing in low density environments, a median FIR luminosity of $10^{11} L_{\odot}$ (which translates to a median star formation rate of around $15 M_{\odot} \mathrm{yr}^{-1}$ ). The FIR luminosity - and therefore the star formation rate - shows little correlation with group richness and scales with the total stellar mass of the system, with little or no dependence on the merger mass ratio. In particular, minor mergers (mass ratios <1:3) are capable of driving strong star formation (between 10 and $173 M_{\odot} \mathrm{yr}^{-1}$ ) and producing systems that are classified as luminous infrared galaxies (LIRGS; 65\% of our LIRGs are minor mergers), with some minor-merging systems being close to the ultra luminous infrared galaxy (ULIRG) limit. Optical emission line ratios indicate that the AGN fraction increases with increasing FIR luminosity, with all ULIRG mergers having some form of AGN activity. Finally, we estimate that the LIRG-to-ULIRG transition along a merger sequence typically takes place over a relatively short timescale of $\sim 160$ Myr.
\end{abstract}

Key words. galaxies: evolution - galaxies: interactions - galaxies: spiral - infrared: galaxies

\section{Introduction}

Mergers are a fundamental feature of our current understanding of the Universe: the standard $\Lambda$ cold dark matter $(\Lambda \mathrm{CDM})$ cosmology (Blumenthal et al. 1984; Freedman et al. 2001; Efstathiou et al. 2002; Pryke et al. 2002; Spergel et al. 2007) is based on a hierarchical structure formation paradigm, in which smaller systems merge to form progressively larger ones (White \& Rees 1978; Searle \& Zinn 1978). Galaxy merging is expected to drive strong star formation episodes (Barnes \& Hernquist 1991; Mihos \& Hernquist 1996), contribute to and regulate the growth of black holes (Kauffmann et al. 2003; Di Matteo et al. 2005; Cox et al. 2006; Schawinski et al. 2009), and produce morphological transformations (Toomre 1977; Mihos \& Hernquist 1996). Given the essential role of mergers in the evolution of the visible Universe, it is important to extend our knowledge of their characteristics beyond the information that we can gather by studying mergers in UV/optical wavelengths alone.

Ultraviolet/optical astronomy, while the cornerstone of our astronomical knowledge, does not provide us with a complete picture of star formation. Short-wavelength photons are affected by absorption and scattering by cold interstellar dust, causing a dimming in the UV/optical light and an apparent reddening of the source (Draine 2003, 2009; Zubko et al. 2004). Indeed, around $50 \%$ of all the energy produced by star formation and active galactic nuclei (AGNs) over cosmic time has been absorbed by molecular clouds and re-emitted in the far-infrared wavebands (3.5-1000 $\mu \mathrm{m}$, e.g. Puget et al. 1996; Fixsen et al. 1998; Pei et al. 1999; Hauser \& Dwek 2001; Dole et al. 2006). Obscuration could be more severe in mergers because of their dustier cores, making it challenging to measure the total star formation activity via the UV/optical wavelengths (Spitzer 1978; Kennicutt 1998; Pei et al. 1999). However, while UV/optical signatures are likely to be affected, the star formation activity can be better studied using far-infrared (FIR) wavelengths, as the peak of emission from cold dust in the star-forming regions lies in the FIR (e.g. Shu et al. 1987; Pei et al. 1999; Chary \& Elbaz 2001).

In recent decades, several studies have looked at the connection between mergers and FIR-bright galaxies. Since the discovery of luminous and ultra-luminous infrared galaxies (LIRGs and ULIRGs), which exhibit FIR luminosities exceeding $10^{11} L_{\odot}$ and $10^{12} L_{\odot}$, respectively (e.g. Soifer et al. 1984; Sanders \& Mirabel 1996), studies have routinely found evidence for morphological disturbances or ongoing mergers in FIR-bright galaxies (Kleinmann \& Keel 1987; Sanders et al. 1987, 1988a,b; Hutchings \& Neff 1987; Vader \& Simon 1987; Sanders \& Mirabel 1996; Clements et al. 1996; Hopkins et al. 2006; Younger et al. 2009; Hwang et al. 2010). While most of these studies have started with a sample of FIR-bright galaxies and found evidence for merger activity within some of them, we approach the infrared properties of mergers from the opposite angle.

We start with a large, visually selected sample of local galaxy mergers $(z<0.1)$ and then explore their infrared properties via their IRAS photometry, thus performing a large blind statistical study of nearby mergers in the FIR. Such a study has been challenging in the past because large unbiased samples of true mergers in the local Universe have been lacking (Darg et al. 2010b,a). Notwithstanding IRAS' low sensitivity compared to newer instruments (e.g. Herschel), an IRAS-based analysis is useful here because the large IRAS beam size of $1.5^{\prime} \times 4.7^{\prime}$ (at $60 \mu \mathrm{m}$, Neugebauer et al. 1984a,b) allows us to study the 

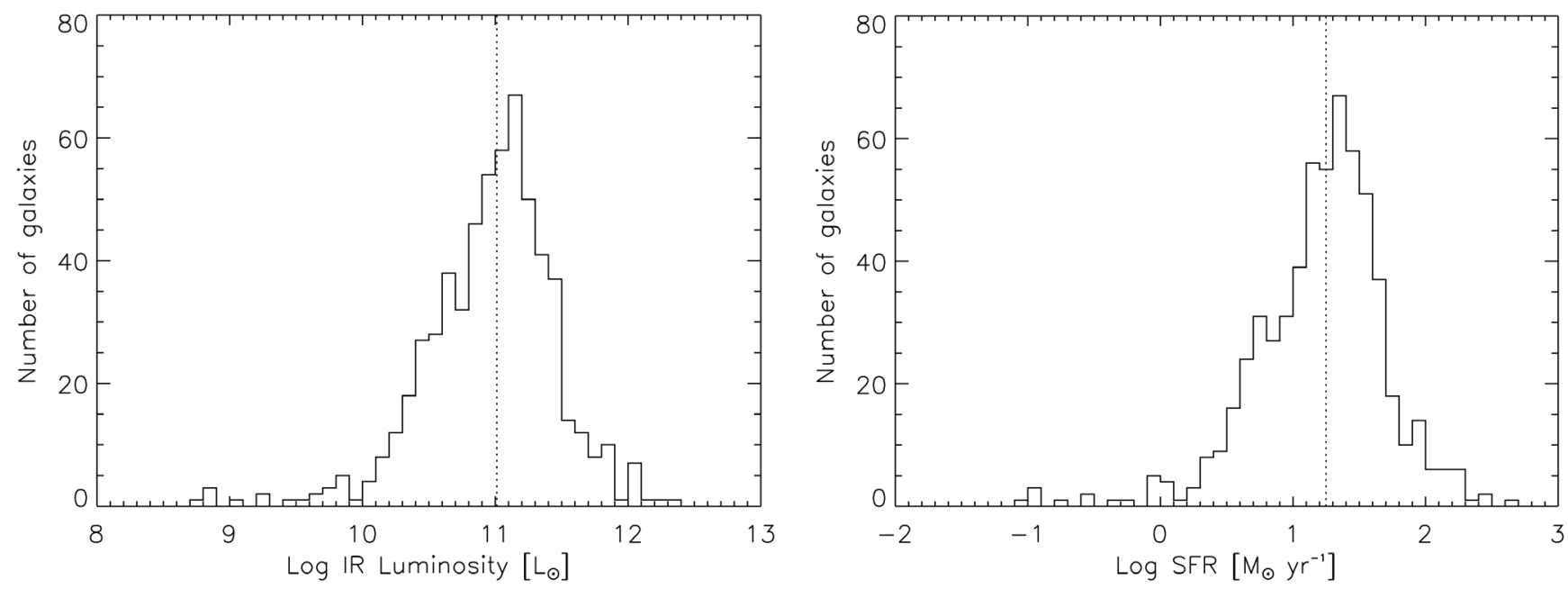

Fig. 1. Distribution of the FIR $(8-1000 \mu \mathrm{m})$ luminosities of the IRAS-detected galaxy mergers $($ left $)$ and the corresponding star formation rates (right), following Eq. (4) in Kennicutt (1998).

entire merging system as a single source. The SDSS mergers are isolated systems, so contamination by nearby sources within the IRAS beam is negligible.

The plan for this paper is as follows. In Sect. 2, we discuss the general properties of the merger sample that underpins this study. In Sect. 3, we study how the star formation in mergers depends on the total mass and mass ratio of the merging systems, while in Sect. 4 we study the impact of local environment on star formation in our mergers. In Sect. 5, we explore the emission line activity in our sample and in Sect. 6, we explore the timescale for LIRGs to transform into ULIRGs along the merger sequence. We summarize our results in Sect. 7. Throughout this paper we adopt the standard cosmological parameters from Spergel et al. (2007) $\left(H_{0}=70 \mathrm{~km} \mathrm{~s}^{-1} \mathrm{Mpc}^{-1}\right.$, $\Omega_{\Lambda}=0.73, \Omega_{\mathrm{M}}=0.27$ ).

\section{The merger sample and basic properties}

Our sample of visually classified mergers was produced via the Galaxy Zoo (GZ) project (Lintott et al. 2008). GZ is uniquely powerful in detecting rare classes of objects like mergers, which can only be reliably selected via direct visual inspection of galaxy images. GZ had enlisted over 500000 volunteers from the general public to morphologically classify (as spiral, elliptical and mergers), through visual inspection, the entire SDSS spectroscopic sample (York et al. 2000; Adelman-McCarthy et al. 2008). This preliminary classification was used by Darg et al. (2010b) to build a merger catalogue of over three thousand objects. The raw parameter $f_{\mathrm{m}}$, called the weighted-merger-vote fraction, is used to select merging galaxies from the whole sample. $f_{\mathrm{m}}$ simply quantifies the probability that a certain image was in fact the image of a merger based on all the classifications of that particular image. The parameter $f_{\mathrm{m}}$ ranges from 0 to 1 so that an object with $f_{\mathrm{m}}=0$ should look nothing like a merger and $f_{\mathrm{m}}=1$ should look unmistakably so. To find mergers within the GZ catalogue, a cut of $0.4<f_{\mathrm{m}}<1.0$ was applied. The reason behind the cut on the weighted-merger-vote fraction was the high occurrence of false positives (which are virtually nonexistent in the interval $f_{\mathrm{m}}>0.6$ ). A second layer of visual inspection, performed by the team, was used to remove any nonmerging systems, visually select an appropriate SDSS object to represent the merging partner, and assign morphologies to the galaxies in each merging system.
The final sample of GZ merger pairs (on which this study is based) contains 3373 objects, unbiased in morphology and local environment and with mass ratios typically between $1: 1$ and $1: 10$ at $z<0.07$. The separation range for these galaxies goes up to $100 \mathrm{kpc}$. Since the merger classification is based on morphological disturbances, the separation peaks at $10 \mathrm{kpc}$ and the number of objects with a separation greater than 20 kpc quickly dwindles. Thus a significant proportion of very early stage mergers are excluded from the catalogue. We refer readers to Darg et al. (2010b) for a complete description of the merger sample.

The GZ merger sample is cross-matched with the Imperial IRAS-FSC Redshift Catalogue (IIFSCz; Wang \& RowanRobinson 2009), a sample of $\sim 60000$ galaxies selected at $60 \mu \mathrm{m}$ from the IRAS Faint Source Catalogue (FSC; Moshir et al. 1992). The IIFSC $z$ catalogue provides FIR fluxes based on the best-fitting infrared templates of Rowan-Robinson et al. (2008). For the flux limit at $60 \mu \mathrm{m}(f(60) \sim 0.36 \mathrm{Jy}), 90$ per cent of sources have spectroscopic redshifts from the SDSS.

Cross-matching the GZ mergers with the IIFSC $z$ yields 606 systems. Our analysis therefore applies to the brightest $18 \%$ of the FIR-detected GZ mergers. In this merger sample, 594 mergers are spiral-spiral merging pairs, with only 12 having an elliptical progenitor. There are 274 LIRGs (45\%) and 10 ULIRGs (2\%) in our sample, with the ULIRGs typically being nearlycoalesced, i.e. in the very final stages of the merger. We note that the Darg et al. merger catalogue is biased against ULIRGs, since they typically do not show two merging nuclei with tidal bridges between them, which are the main criteria for a galaxy pair to be defined as a merger in GZ (e.g. the ULIRG fraction from the GOALS survey is 10\%, Armus et al. 2009).

The $L_{\text {FIR }}$ in our IRAS-detected mergers ranges from around $10^{9} L_{\odot}$ to over $10^{12.3} L_{\odot}$, with a median of $\sim 10^{11} L_{\odot}$, which corresponds to a star formation rate (SFR) of $15 M_{\odot} \mathrm{yr}^{-1}$ (see Fig. 1), calculated using Kennicutt (1998):

$$
\operatorname{SFR}_{\mathrm{FIR}}\left(M_{\odot} \mathrm{yr}^{-1}\right)=4.5 \times 10^{-44} L_{\mathrm{FIR}}\left(\mathrm{erg} \mathrm{s}^{-1}\right) .
$$

To compare the properties of our IRAS-detected mergers to the general galaxy population in our subsequent analysis, we construct a control sample of 2300 relaxed (i.e. non-merging) galaxies from Galaxy Zoo and the IIFSC $z$, selected to have the same redshift and $r$-band magnitude ranges as the mergers. Most of the galaxies in our sample are spirals (2250) with a small fraction 


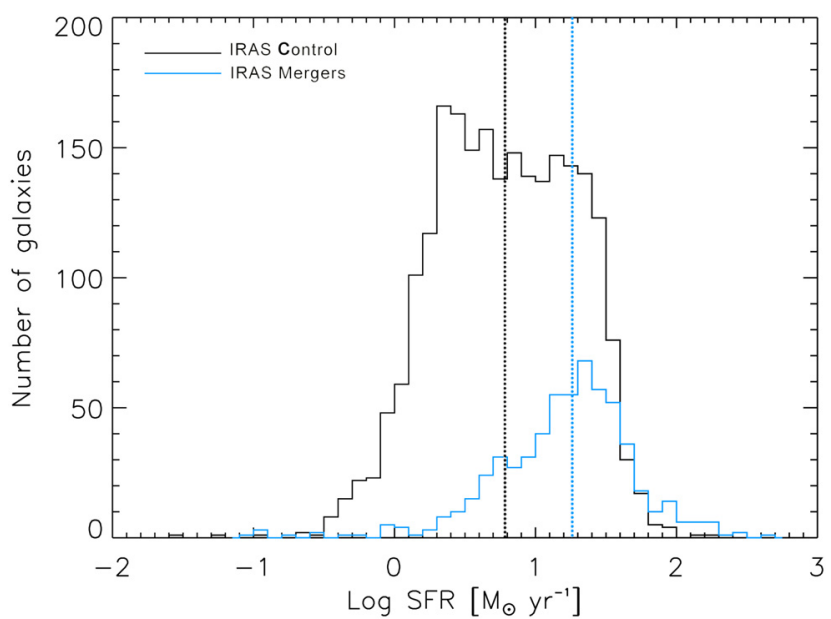

Fig. 2. Distribution of star formation rates for the IRAS-detected mergers and for a control sample of non-merging galaxies detected by IRAS. Median values are indicated using the dotted vertical lines.

of ellipticals (50) to keep a similar proportion to the morphology in the IRAS mergers (49.5 spirals per elliptical).

\section{Dependence of SFR on system mass and mass ratio}

We begin by studying how the SFRs of mergers are influenced by their total stellar mass and the merger mass ratio. The stellar mass was calculated by Darg et al. (2010b) by fitting each galaxy's SDSS photometry to a library of photometries produced by a variety of two-component star formation histories, where the older burst is taken as a simple stellar population while the more recent burst is modelled by an exponential to take into account the extended star formation history of mergers. The library of SEDs are generated using the Maraston (1998, 2005) stellar models. Both components have stellar populations with variable age with fixed solar metallicity and Salpeter IMF (Salpeter 1955). Dust is implemented using a Calzetti et al. (2000) law with an $E(B-V)$ varying between 0 and 0.6. The uncertainty on the masses for the GZ merger sample is 0.2 dex (Darg et al. 2010a,b)

In Fig. 3 we study the dependence of the SFR on the total (stellar) mass of the system (left panel) and on the merger mass ratio (right panel).

The SFR is positively correlated with the total stellar mass of the system, with LIRGs and ULIRGs residing mostly at the higher end of the mass spectrum. While the positive correlation exists both for the mergers and the control sample, the mergers show a largely mass-independent enhancement in SFR of 0.5 dex. The enhancement at the low mass end of the distribution is within the one-sigma error in the fit. There is a wide spread in the SFR of the mergers at the low mass end and a proportionally smaller number density compared to the higher end of the distribution. The linear fit for the distributions are

$\log (S F R)=0.5( \pm 1.2) \log ($ Mass $)-4.1$

for the mergers, and

$\log (S F R)=0.8( \pm 1.3) \log ($ Mass $)-8.1$

for the control sample. The control sample fit is consistent with the SFR-Mass relation for SDSS galaxies found in Elbaz et al. (2007) and in Willett et al. (2015), which also reported a SFR enhancement in the local merger population.

We find no apparent dependence between the mass ratio of the merger and the SFR in the merging galaxies. As an ulterior check we compare merger systems within the same total stellar mass range: we plot the mass ratio versus the total stellar mass and the mass ratio versus the specific SFR. No correlation was found in those plots and they hence have not been included. Minor mergers (those with mass ratios less than 1:3) seem capable of producing IR-bright systems, including LIRGs and systems that are close to the ULIRG limit $\left(L>10^{12} l_{\odot}\right)$. Our analysis, therefore, suggests that major mergers are not the only process that can trigger strong star formation episodes (between 10 and $173 M_{\odot} \mathrm{yr}^{-1}$ ), somewhat in contradiction with the wider literature (Di Matteo et al. 2005; Cox et al. 2006). In particular, minor mergers appear to play an influential role (65\% of LIRGs are minor mergers) in triggering such strong star formation episodes, in agreement with the findings of recent observational work (Scudder et al. 2012; Kaviraj 2014a,b).

Overall, we find that the star formation activity in mergers is correlated more strongly with the total stellar mass of the merging system, with the system mass ratio being of negligible importance.

\section{Dependence of SFR on local environment}

In Fig. 4 we explore the dependence of the merger SFR on local environment. We use the Yang et al. environment catalogue (Yang et al. 2007) to estimate the environment of our merging systems. Yang et al. estimate the dark matter halo mass of individual galaxies, which can be used as a proxy for the local environment of the galaxy. Typically, haloes with masses lower than $10^{13} M_{\odot}$ are considered to be the field, haloes with masses between $10^{13} M_{\odot}$ and $10^{14} M_{\odot}$ are considered to be in group-like environments, while haloes with masses greater than $10^{14} M_{\odot}$ host clusters (Kaviraj et al. 2009).

Across the range of environments sampled by our mergers, we do not find a strong correlation between the SFR in merging galaxies and their local environment. The relationship between SFR and environment in the mergers is similar to that in the control sample, except that the mergers show an enhancement in SFR of around 0.5 dex, largely irrespective of environment. In agreement with previous studies (e.g. Hwang et al. 2010; Darg et al. 2010b; Carpineti et al. 2012), we find that, like the general merger population, the IRAS-detected mergers tend to favour lower-density environments. Most of the IRASdetected mergers are found in groups and the field (95\% of the sample favours such low-density environments), with ULIRGs exclusively inhabiting the field.

\section{Emission line activity}

We use the nuclear activity classification from Darg et al. (2010b) to investigate the ionization mechanisms in the IRAS-detected mergers and probe the connection between AGN activity and FIR luminosity. The classification was done by performing a BPT analysis (Baldwin et al. 1981) on the full GZ merger catalogue, using optical emission-line ratios from the SDSS. The line ratios used were $[\mathrm{OIII}] / \mathrm{H} \beta,[\mathrm{NII}] / \mathrm{H} \alpha,[\mathrm{SII}] / \mathrm{H} \alpha$ and $[\mathrm{OI}] / \mathrm{H} \alpha$, to obtain three reddening insensitive diagnostic diagrams following Veilleux \& Osterbrock (1987). Darg et al. (2010b) used the demarcation lines from Kauffmann et al. (2003), Kewley et al. (2006) to separate galaxies into ones that 

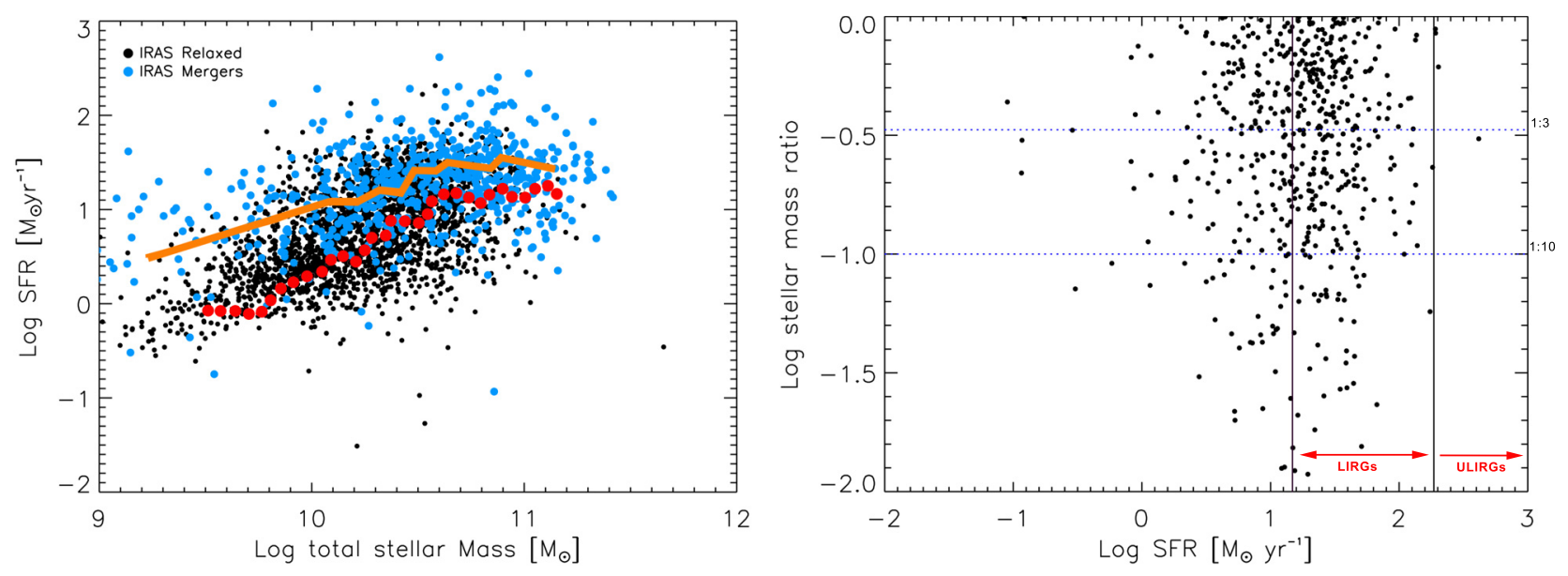

Fig. 3. Left: star formation rate versus total stellar mass for mergers and the control sample of relaxed non-merger galaxies. Right: star formation rate in mergers plotted against merger mass ratio. Mass ratios of 1:3 and 1:10 are indicated using the dotted horizontal lines. From left to right the solid vertical lines represent the star formation rates for LIRGs and ULIRGs. There is no significant trend between SFR and mass ratio. It is also important to note that minor mergers play a significant role in the formation of LIRGs. The left plot also shows a progressive one-sigma fit to the mergers (solid orange) and control sample (dashed red).

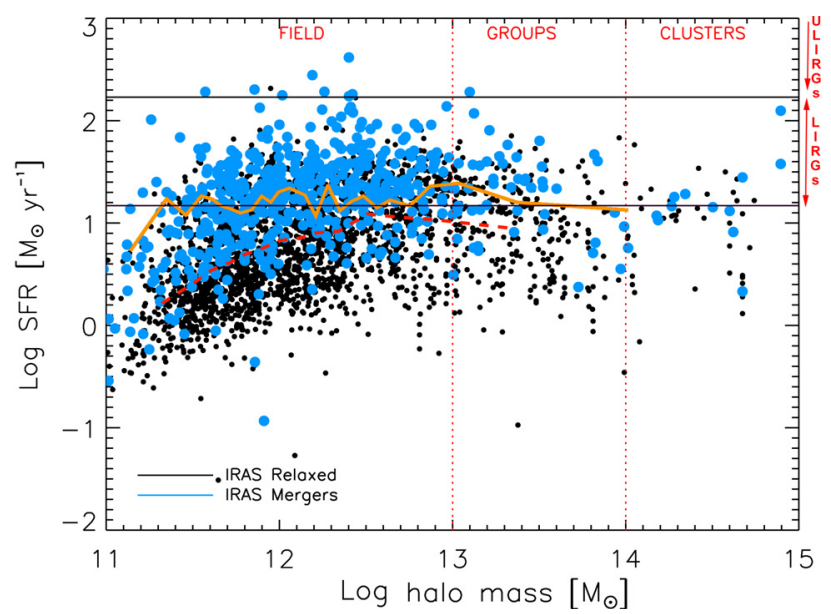

Fig. 4. Star formation rate (SFR) of the IRAS-detected mergers (blue) and the LTG control sample (black) plotted against local environment. The plot also shows a progressive one-sigma fit to the mergers (solid orange) and control sample (dashed red).

are star-forming, AGN, LINERs, or SF/AGN (i.e. contain both star formation and AGN), using a signal-to-noise $(\mathrm{S} / \mathrm{N})$ threshold of 3 in all lines. Galaxies which do not have $S / N>3$ in $\mathrm{H} \alpha, \mathrm{H} \beta$, NII, OIII lines are classified as quiescent. The ratios were computed using the GANDALF code (Sarzi et al. 2006). We used the same approach to classify the ionization mechanisms for the control sample.

In Fig. 5, we split the IRAS-detected mergers into these emission-line categories and study how they change with FIR luminosity. We also perform an equivalent analysis for our control sample of galaxies from the IIFSC $z$. The majority $(71 \%)$ of the IRAS-detected mergers are classified as star-forming, with a significant minority (35\%) hosting an AGN. Mergers that are LIRGs exhibit a slightly higher incidence of AGN with respect to the general IRAS-detected merger population and the control sample. However, in mergers that are ULIRGs the ionization appears to be dominated by the AGN. Six out of the ten ULIRGs in our merger sample have a Seyfert-type AGN, while the other two are classified as LINERs. This result appears consistent with the recent literature which suggests that
AGN become active in the late stages of a merger (Schawinski et al. 2007; Darg et al. 2010a; Wild et al. 2010; Kaviraj et al. 2011; Carpineti et al. 2012) and also with the findings of past studies (Sanders \& Mirabel 1996; Clements et al. 1996; Risaliti et al. 2000; Hopkins et al. 2006; Chakrabarti et al. 2007; Younger et al. 2009; Treister et al. 2009, 2010; Iwasawa et al. 2011) which suggest that AGN play an important role in the formation and evolution of ULIRGs - at least 50\% (and up to 75\%) of the ULIRGs explored in past studies show an AGN signature. Studies in Mid-IR in the past few years have found similar results, highlighted the more likely dominance SF in coalescing mergers (Alonso-Herrero et al. 2010; Pereira-Santaella et al. 2010), showed a similar increase in AGN to SF activity in late stage mergers (Petric et al. 2011), and also found an increase in composite SF/AGN objects (Stierwalt et al. 2013).

\section{Timescale of LIRG to ULIRG transformation along a merger sequence}

While our sample of ULIRGs is small, we find a clear morphological segregation between LIRGs and ULIRGs in our sample. In Fig. 6 we present typical SDSS images of ULIRGs in our sample. Half the ULIRGs in our sample are in an advanced merging state (the projected distance between their cores is less than $4 \mathrm{kpc}$ in all cases), while the other half have already coalesced (i.e. it is not possible to resolve two cores in the SDSS images). The angular resolution of the SDSS is $\sim 1.3^{\prime \prime}$ (Bramich \& Freudling 2012). In comparison, $98 \%$ of LIRGs have a core separation greater than $4 \mathrm{kpc}$ in the redshift limit of our sample $(z=0.05$ and $z=0.1) ; 4 \mathrm{kpc}$ equates to $4.1^{\prime \prime}$ and $2.2^{\prime \prime}$, respectively, much greater than the SDSS limit (left panel of Fig. 7). Assuming that an evolutionary transition occurs from LIRG to ULIRG as the merger progresses and star formation increases (Sanders \& Mirabel 1996; Clements et al. 1996; Dasyra et al. 2006), it is instructive to explore how quickly the LIRGto-ULIRG transition is likely to take place along the merger sequence.

An estimate - albeit crude - can be derived for this coalescence timescale using the typical separations of the LIRGs in our merger sample and the velocity dispersion of the groups that 


\section{$\square$ Quiescent $\square$ SF SF/AGN $\square$ AGN $\square$ LINER}

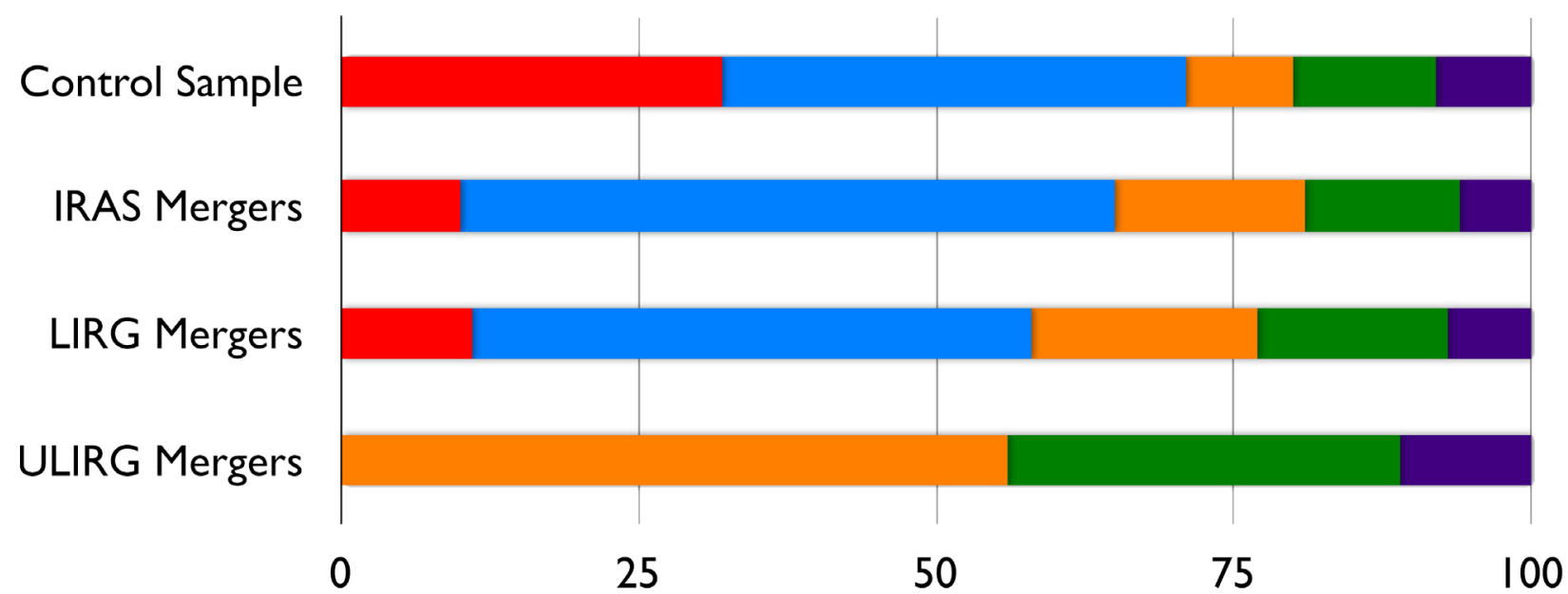

Fig. 5. Emission-line classes for the control sample, IRAS-detected mergers and the subsets of the IRAS-detected mergers that are classified as LIRGs and ULIRGs.
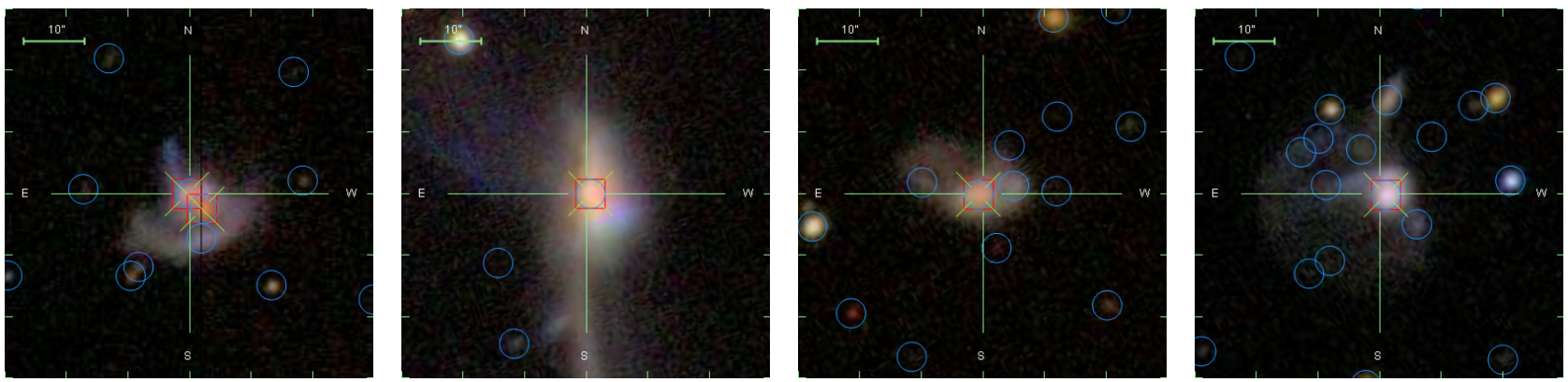

Fig. 6. SDSS images of 4 typical ULIRGs in our sample: in order IRAS F12112+0305, Mrk 273, IRAS F15250+3608, IRAS F16133+2107.
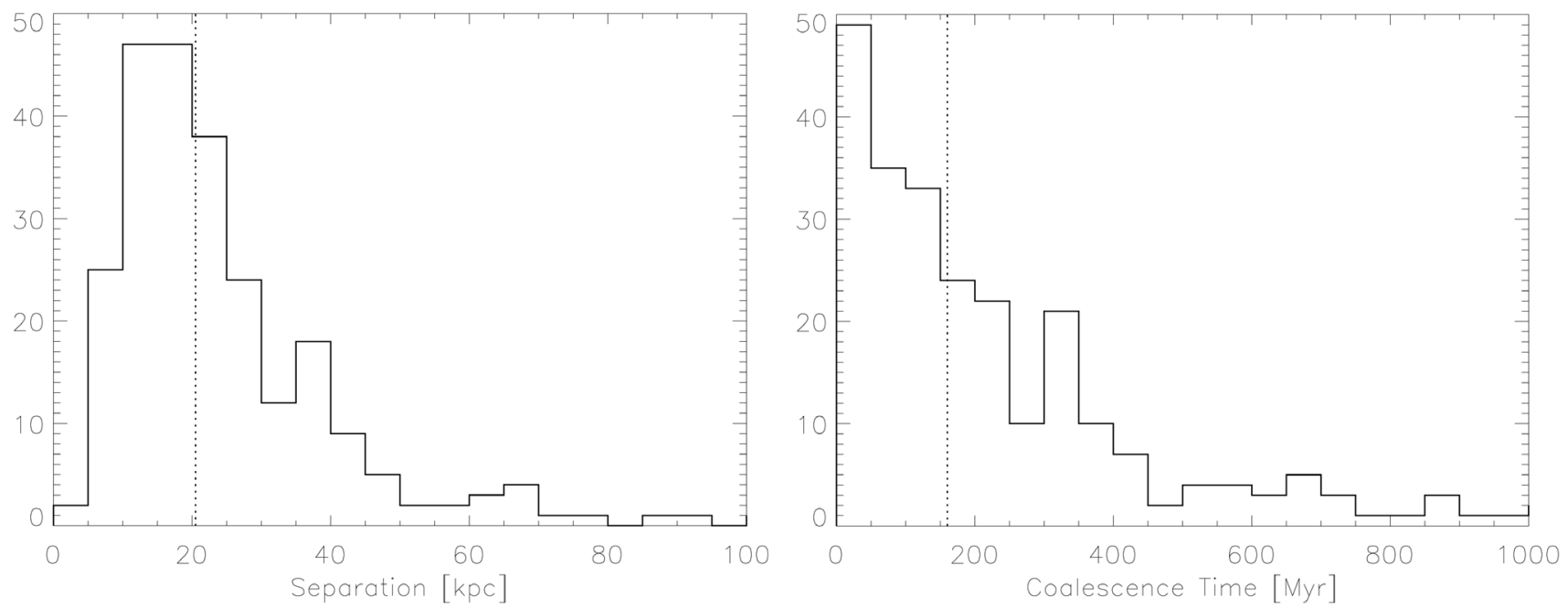

Fig. 7. Projected separation (left) and derived coalescence timescales (right) of merging LIRGs in our sample. See text for more details.

they inhabit (which is a measure of the typical relative velocities of objects in a group).

Keeping in mind that there may be a selection bias in the merger sample against ULIRGs, we first check whether the ULIRGs in our merger sample are representative of ULIRGs in general. We do so by extracting all 121 ULIRGs in the IIFSC $z$ at $z<0.1$ and inspecting their SDSS images. We find that in this general ULIRG sample, more than $90 \%$ are indeed postmerger systems with a single core and significant tidal debris, very similar to the ULIRGs in the merger sample. The postmerger nature of ULIRGs in the Darg et al. sample thus appears to be a typical characteristic of these systems, as has been suggested by the past literature (Sanders \& Mirabel 1996; Clements et al. 1996; Dasyra et al. 2006).

In the bottom plot of Fig. 7, we estimate a timescale for LIRGs to transform into ULIRGs along the merger sequence. 
For most LIRGs we can only construct a projected distance because fibre collisions mean that typically only one progenitor has an SDSS redshift (so the line-of-sight separation cannot be computed). Nevertheless, 32 merging LIRGs do have redshifts for both progenitors. In these systems we compare the projected separation and the true separation, to gauge the error in the galaxy separations produced by using only projected separations. We find that the true separations in this subsample of LIRGs are only around $8 \%$ larger than the projected separations. In other words, projected separations seem to be reasonably representative of true separations, at least in the subsample of our merging LIRGs in which both progenitors have an SDSS redshift. This is not unexpected, since these are merging galaxies which, by definition, must be close to each other (i.e. they must be virtually at the same redshift). Assuming this is true for merging LIRGs in general, we assume the projected separations to be reasonable estimates for the true separations in all merging LIRGs in our sample.

To estimate a coalescence timescale we require an estimate for the relative velocities of the individual galaxies. While we do not have the information to measure either the relative transverse or line-of-sight velocities (except in the 32 systems above where we can only measure the relative line-of-sight velocities), we use the mean velocity dispersion of SDSS groups $(258 \pm 7)$ from Nurmi et al. (2013). To estimate the timescale we use the following equation

$$
T_{\text {coal }}=M_{\text {ratio }} \times 2 \pi \times \frac{r}{\sigma},
$$

where $r$ is the separation, $\sigma$ is the velocity dispersion, and $M_{\text {ratio }}$ the mass ratio of the two galaxies. The timescales are plotted in the right panel of Fig. 7. From the distribution of coalescence timescales, we estimate that the timescale for the LIRG to ULIRG transformation is likely to be a few hundred Myr $(160 \pm$ $56 \mathrm{Myr}$ ). Our empirically-determined values and the range of the timescale appear to be in reasonably good agreement with previous surveys (Murphy et al. 1996; Haan et al. 2011) as well as $N$-body/SPH merger simulations (see e.g. Torrey et al. 2012a,b) which find coalescence timescales of $200 \mathrm{Myr}$. The low values of the timescales indicate that merging LIRGs turn into ULIRGs over reasonably short timescales, comparable to the dynamical timescales of star formation in these systems.

\section{Summary}

We have combined a sample of $\sim 3000$ visually selected local mergers from the Galaxy Zoo project with the Imperial IRAS Faint Source Redshift Catalogue, to perform a large blind FIR study of the local merger population. 18\% of the GZ mergers are detected by IRAS. Our results therefore describe the brightest $18 \%$ of merging systems in terms of FIR luminosity (and therefore SFR).

The IRAS-detected mergers are overwhelmingly (98\%) comprised of spiral-spiral systems, with a median FIR luminosity of $10^{11} L_{\odot}$ and a median SFR of around $15 M_{\odot} \mathrm{yr}^{-1}$. The SFR of the IRAS-detected mergers are offset from the general IRASdetected galaxy population by 0.5 dex. While the merger SFR correlates with the total mass of the merging system, we find very little dependence on the merger mass ratio. Indeed, 65\% of the systems with LIRG luminosities (and possibly some lowluminosity ULIRGs) are hosted by minor mergers (mass ratios lower than 1:3). This suggests that strong star formation (between 10 and $173 M_{\odot} \mathrm{yr}^{-1}$ ) can be triggered by the minor-merger process, in good agreement with the recent literature.
While they reside in low-density environments, the merger SFR does not show a strong dependence on local density. An optical emission-line ratio analysis indicates that the contribution of AGN to the gas ionization increases with increasing FIR luminosity. The AGN fraction increases dramatically for ULIRGs - almost all ULIRGs show emission-line diagnostics indicative of an AGN component, despite being strongly starforming systems.

A particular characteristic of ULIRGs is that they are all in advance merger stage: either the nuclei are nearly coalesced (with a separation $<4 \mathrm{kpc}$ ) or they are already in a coalesced post merger phase. Assuming that the (non-coalesced) merging LIRGs are the parent population for ULIRGs, we have estimated that galaxies transition from being LIRGs to ULIRGs along the merger sequence over a reasonably short timescale $(\sim 160 \pm 56 \mathrm{Myr})$.

Acknowledgements. The authors thank the anonymous referee for comments and suggestions that greatly improved this paper. The data in this paper are the result of the efforts of the Galaxy Zoo volunteers, without whom none of this work would be possible. Their efforts are individually acknowledged at http:// authors.galaxyzoo.org. Galaxy Zoo was supported by The Leverhulme Trust. SK acknowledges a Senior Research Fellowship from Worcester College Oxford.

\section{References}

Adelman-McCarthy, J. K., Agüeros, M. A., Allam, S. S., et al. 2008, ApJS, 175, 297

Alonso-Herrero, A., Pereira-Santaella, M., Rieke, G. H., et al. 2010, Adv. Space Res., 45, 99

Armus, L., Mazzarella, J. M., Evans, A. S., et al. 2009, PASP, 121, 559

Baldwin, J. A., Phillips, M. M., \& Terlevich, R. 1981, PASP, 93, 5

Barnes, J. E., \& Hernquist, L. E. 1991, ApJ, 370, L65

Blumenthal, G. R., Faber, S. M., Primack, J. R., \& Rees, M. J. 1984, Nature, 311,517

Bramich, D. M., \& Freudling, W. 2012, MNRAS, 424, 1584

Calzetti, D., Armus, L., Bohlin, R. C., et al. 2000, ApJ, 533, 682

Carpineti, A., Kaviraj, S., Darg, D., et al. 2012, MNRAS, 420, 2139

Chakrabarti, S., Cox, T. J., Hernquist, L., et al. 2007, ApJ, 658, 840

Chary, R., \& Elbaz, D. 2001, ApJ, 556, 562

Clements, D. L., Sutherland, W. J., McMahon, R. G., \& Saunders, W. 1996, MNRAS, 279, 477

Cox, T. J., Dutta, S. N., Di Matteo, T., et al. 2006, ApJ, 650, 791

Darg, D. W., Kaviraj, S., Lintott, C. J., et al. 2010a, MNRAS, 401, 1552

Darg, D. W., Kaviraj, S., Lintott, C. J., et al. 2010b, MNRAS, 401, 1043

Dasyra, K. M., Tacconi, L. J., Davies, R. I., et al. 2006, ApJ, 638, 745

Di Matteo, T., Springel, V., \& Hernquist, L. 2005, Nature, 433, 604

Dole, H., Lagache, G., Puget, J.-L., et al. 2006, A\&A, 451, 417

Draine, B. T. 2003, ARA\&A, 41, 241

Draine, B. T. 2009, in Cosmic Dust - Near and Far, eds. T. Henning, E. Grün, \& J. Steinacker, ASP Conf. Ser., 414, 453

Efstathiou, G., Moody, S., Peacock, J. A., et al. 2002, MNRAS, 330, L29

Elbaz, D., Daddi, E., Le Borgne, D., et al. 2007, A\&A, 468, 33

Fixsen, D. J., Dwek, E., Mather, J. C., Bennett, C. L., \& Shafer, R. A. 1998, ApJ, 508, 123

Freedman, W. L., Madore, B. F., Gibson, B. K., et al. 2001, ApJ, 553, 47

Haan, S., Surace, J. A., Armus, L., et al. 2011, AJ, 141, 100

Hauser, M. G., \& Dwek, E. 2001, ARA\&A, 39, 249

Hopkins, P. F., Somerville, R. S., Hernquist, L., et al. 2006, ApJ, 652, 864

Hutchings, J. B., \& Neff, S. G. 1987, AJ, 93, 14

Hwang, H. S., Elbaz, D., Lee, J. C., et al. 2010, A\&A, 522, A33

Iwasawa, K., Sanders, D. B., Teng, S. H., et al. 2011, A\&A, 529, A106

Kauffmann, G., Heckman, T. M., Tremonti, C., et al. 2003, MNRAS, 346, 1055

Kaviraj, S. 2014a, MNRAS, 440, 2944

Kaviraj, S. 2014b, MNRAS, 437, L41

Kaviraj, S., Peirani, S., Khochfar, S., Silk, J., \& Kay, S. 2009, MNRAS, 394, 1713

Kaviraj, S., Schawinski, K., Silk, J., \& Shabala, S. S. 2011, MNRAS, 415, 3798

Kennicutt, Jr., R. C. 1998, ARA\&A, 36, 189

Kewley, L. J., Groves, B., Kauffmann, G., \& Heckman, T. 2006, MNRAS, 372, 961 
A. Carpineti et al.: An infrared study of local galaxy mergers

Kleinmann, S., \& Keel, W. 1987, Star Formation in Galaxies (C.J. LonsdalePersson, Washington DC: US Govt. Print. Off.)

Lintott, C. J., Schawinski, K., Slosar, A., et al. 2008, MNRAS, 389, 1179

Maraston, C. 1998, MNRAS, 300, 872

Maraston, C. 2005, MNRAS, 362, 799

Mihos, J. C., \& Hernquist, L. 1996, ApJ, 464, 641

Moshir, M., Kopman, G., \& Conrow, T. A. O. 1992, IRAS Faint Source Survey, Explanatory supplement version 2 (California Institute of Technology)

Murphy, Jr., T. W., Armus, L., Matthews, K., et al. 1996, AJ, 111, 1025

Neugebauer, G., Habing, H. J., van Duinen, R., et al. 1984a, ApJ, 278, L1

Neugebauer, G., Soifer, B. T., Miley, G., et al. 1984b, ApJ, 278, L83

Nurmi, P., Heinämäki, P., Sepp, T., et al. 2013, MNRAS, 436, 380

Pei, Y. C., Fall, S. M., \& Hauser, M. G. 1999, ApJ, 522, 604

Pereira-Santaella, M., Alonso-Herrero, A., Rieke, G. H., et al. 2010, ApJS, 188, 447

Petric, A. O., Armus, L., Howell, J., et al. 2011, ApJ, 730, 28

Pryke, C., Halverson, N. W., Leitch, E. M., et al. 2002, ApJ, 568, 46

Puget, J.-L., Abergel, A., Bernard, J.-P., et al. 1996, A\&A, 308, L5

Risaliti, G., Gilli, R., Maiolino, R., \& Salvati, M. 2000, A\&A, 357, 13

Rowan-Robinson, M., Babbedge, T., Oliver, S., et al. 2008, MNRAS, 386, 697

Salpeter, E. E. 1955, ApJ, 121, 161

Sanders, D. B., \& Mirabel, I. F. 1996, ARA\&A, 34, 749

Sanders, D. B., Scoville, N. Z., Soifer, B. T., Young, J. S., \& Danielson, G. E. 1987, ApJ, 312, L5

Sanders, D. B., Soifer, B. T., Elias, J. H., et al. 1988a, ApJ, 325, 74

Sanders, D. B., Soifer, B. T., Elias, J. H., Neugebauer, G., \& Matthews, K. 1988b, ApJ, 328, L35

Sarzi, M., Falcón-Barroso, J., Davies, R. L., et al. 2006, MNRAS, 366, 1151

Schawinski, K., Kaviraj, S., Khochfar, S., et al. 2007, ApJS, 173, 512
Schawinski, K., Virani, S., Simmons, B., et al. 2009, ApJ, 692, L19

Scudder, J. M., Ellison, S. L., Torrey, P., Patton, D. R., \& Mendel, J. T. 2012, MNRAS, 426, 549

Searle, L., \& Zinn, R. 1978, ApJ, 225, 357

Shu, F. H., Adams, F. C., \& Lizano, S. 1987, ARA\&A, 25, 23

Soifer, B. T., Rowan-Robinson, M., Houck, J. R., et al. 1984, ApJ, 278, L71

Spergel, D. N., Bean, R., Doré, O., et al. 2007, ApJS, 170, 377

Spitzer, L. 1978, Physical processes in the interstellar medium (Wiley-VCH)

Stierwalt, S., Armus, L., Surace, J. A., et al. 2013, ApJS, 206, 1

Toomre, A. 1977, in Evolution of Galaxies and Stellar Populations, eds. B. M. Tinsley, \& R. B. G. Larson, D. Campbell, 401

Torrey, P., Cox, T. J., Kewley, L., \& Hernquist, L. 2012a, ApJ, 746, 108

Torrey, P., Vogelsberger, M., Sijacki, D., Springel, V., \& Hernquist, L. 2012b, MNRAS, 427, 2224

Treister, E., Cardamone, C. N., Schawinski, K., et al. 2009, ApJ, 706, 535

Treister, E., Natarajan, P., Sanders, D. B., et al. 2010, Science, 328, 600

Vader, J. P., \& Simon, M. 1987, Nature, 327, 304

Veilleux, S., \& Osterbrock, D. E. 1987, in NASA Conf. Publ. 2466, ed. C. J. Lonsdale Persson, 737

Wang, L., \& Rowan-Robinson, M. 2009, MNRAS, 398, 109

White, S. D. M., \& Rees, M. J. 1978, MNRAS, 183, 341

Wild, V., Heckman, T., \& Charlot, S. 2010, MNRAS, 405, 933

Willett, K. W., Schawinski, K., Simmons, B. D., et al. 2015, MNRAS, submitted [arXiv: 1502.03444]

Yang, X., Mo, H. J., van den Bosch, F. C., et al. 2007, ApJ, 671, 153

York, D. G., Adelman, J., Anderson, Jr., J. E., et al. 2000, AJ, 120, 1579

Younger, J. D., Hayward, C. C., Narayanan, D., et al. 2009, MNRAS, 396, L66

Zubko, V., Dwek, E., \& Arendt, R. G. 2004, ApJS, 152, 211 